\title{
Metalens-based Particle Routing in Continuous-flow Microchannels
}

\author{
Shengqi Yin, Fei He, Nicolas G Green \& Xu Fang* \\ School of Electronics and Computer Science, University of Southampton, Southampton SO17 1BJ, UK \\ *x.fang@soton.ac.uk
}

We demonstrate dielectric metalenses with phase profiles that respond to changes in two input control light beams, resulting in a steerable focal line. We further show their application of particle routing in continuousflow microchannels.

Nanophotonic devices, ranging from plasmonic thin films to dielectric metamaterials, have demonstrated capability of advanced optical manipulation in microfluidic channels (e.g. [1,2]). Metalenses in particular have attracted great attention from the research community recently, as they enable light focusing in the far field via near-field nano-engineering. Here we demonstrate, via numerical simulation, metalenses with tunable Bessel and parabolic phase profiles that can be used for microfluidic particle routing.
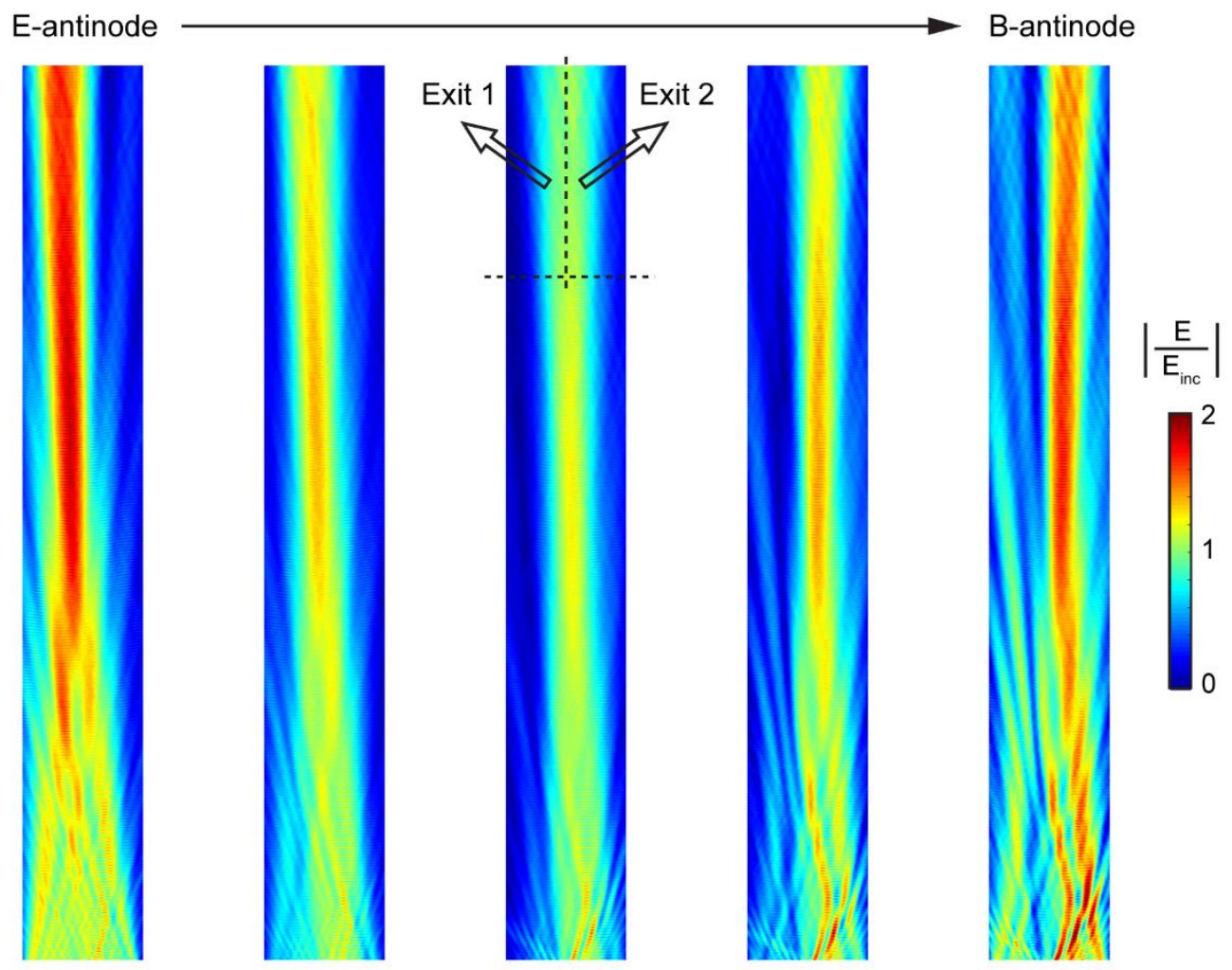

Fig. 1 Steering of light focus inside the water channel (area above the horizontal dashed line) and the intermediate PDMS layer (below the line), enabled by tuning the local light field at the position of an axicon metalens $(\sim 2 \mu \mathrm{m}$ beneath the bottom of each panel). The local field changes gradually from the E-antinode (left most panel) to the B-antinode (right most panel), shifting the light focus across the middle line (the vertical dashed line) of the microchannel and resulting in particle routing.

Figure 1 shows the output electric field from an axicon metalens, where the focus is steerable in the plane normal to the direction of fluid flow. The metalens approximates a cylindrical glass lens, constituting identical lines of Si nanopillars. In each line of such metalenses there are $\sim 20$ nanopillars, which collectively form either a $\mathrm{V}$-shaped phase profile (in the case of an axicon metalens as shown here) or a parabolic phase profile (in the case of a conventional metalens) under the illumination of a single beam of incident light. We further use a second light beam to create a series of different local electromagnetic fields (e.g. the E- and the B-antinodes) at the metalens. The relative strength and phase of multipole Mie resonances of the Si nanopillars change in response to the local driving field, rotating both the total phase profile and the light focus inside the microchannel.

\section{References}

[1] S. Yin, F. He, N. G. Green, and X. Fang, " Nanoparticle trapping and routing on plasmonic nanorails in a microfluidic channel," Optics Express 28, 1357 (2020).

[2] S. Yin, F. He, W. Kubo, Q. Wang, J. Frame. N. G. Green, and X. Fang, "Coherently tunable metalens tweezers for optofluidic particle routing," Optics Express 28, 38949 (2020). 